\title{
Molecular insight into apoptosis mediated by flavones in cancer (Review)
}

\author{
RITU RAINA, ARIF HUSSAIN and RIA SHARMA \\ School of Life Sciences, Manipal Academy of Higher Education, P.O. Box 345050 Dubai, United Arab Emirates
}

Received March 13, 2020; Accepted April 16, 2020

DOI:10.3892/wasj.2020.47

\begin{abstract}
Apoptosis, a vital component of normal cell growth and development is characterized by diverse morphological characteristics. The evasion of apoptosis is an important hallmark of cancer; thus, the ability to induce apoptosis and suppress cell growth is a promising therapeutic approach and a highly active area of cancer research. Although chemotherapeutics can be used to achieve this, their use is associated with high levels of toxicity. Hence, focus is being shifted towards plant-based compounds due to their low toxicity, easy availability and affordability. Flavonoids, a subclass of polyphenols, have attracted attention as potential anticancer candidates due to their ability to induce apoptosis and thus, to reduce cellular proliferation specifically in cancer cells. Several pro- and anti-apoptotic proteins have been identified, although their exact modes of action have yet to be elucidated. Therefore, research continues to focus on the pathways followed by key proteins involved in cell cycle progression and apoptosis. This arena of apoptosis research has been moving forward at a remarkably rapid pace. The aim of the present review was to provide an overall summary of the presently available data on the modulation of the process of apoptosis by flavones in different types of cancer.
\end{abstract}

Correspondence to: Dr Arif Hussain, School of Life Sciences, Manipal Academy of Higher Education, P.O. Box 345050 Dubai, United Arab Emirates

E-mail: dr.arifhussain@yahoo.co.in

Abbreviations: BAX, Bcl-2-associated X protein; Bcl-2, B-cell lymphoma 2; Bcl-xL, B-cell lymphoma-extra large; Mcl-1, myeloid cell leukemia 1; PARP, poly(ADP-ribose) polymerase; AOM, azoxymethane; TRAMP, transgenic adenocarcinoma of mouse prostate; AMPK, AMP-activated protein kinase; STAT, signal transducer and activator of transcription; mTOR, mammalian target of rapamycin; NF- $\kappa \mathrm{B}$, nuclear factor $\kappa \mathrm{B}$

Key words: apoptosis, apigenin, baicalein, chrysin, luteolin, flavones

\section{Contents}

1. Introduction

2. Chemoprevention

3. Role of diet in the prevention of carcinogenesis

4. Flavones and apoptosis

5. Conclusion and future prospects

\section{Introduction}

Cancer is a complex, multifactorial disease that is characterized by uncontrolled cellular proliferation, leading to tumor development. It develops as a result of genetic and epigenetic alterations in the genome caused by errors in the replication of DNA or interactions with exogenous agents, such as radiation and chemical carcinogens. The accumulation of such tumor-promoting modulations in the DNA of cells endows them with the ability to evade programmed cell death, a process known as apoptosis, which is a crucial homeostatic mechanism that regulates cell turnover and maintains cell populations in tissues. The types of genes involved in the development of carcinogenesis include tumor suppressor genes (e.g., p53 and Rb), oncogenes (e.g., myc and Ras) and DNA repair genes (P53 and BRCA1) (1). Cancer is being extensively researched as it is a leading cause of mortality worldwide. It accounted for an estimated 9.6 million deaths only in 2018 (2). Studies that have examined the risk factors associated with the incidence of cancer have found that a high body mass index (BMI, a sedentary lifestyle, smoking, alcohol consumption and a low consumption of plant-based foods, are top risk factors for cancer development, among others (2-5). The disease is conventionally treated using surgery, radiation therapy and chemotherapy (or a combination of these) depending on the cancer type, its stage and its location. A great challenge in the pursuit for effective cancer treatment is the genotypic and phenotypic heterogeneity of tumor cells. Thus far, the cytotoxic agents that have been used in chemotherapy act rapidly, dividing abnormal cells by inducing cell cycle disruption or mitotic division failure, effectively killing tumor cells, while also killing normal cells with a high rate of turnover; this results in adverse side-effects, which include depression of the bone marrow, aplastic anemia, diarrhea, vomiting, alopecia etc. (6). To this effect, the greatest limitations of chemotherapy are its non-selective cytotoxic effects, its lack of efficiency and 
the associated financial cost. Therefore, the need for the development of agents, not only with a high efficacy and specificity, but also with a low toxicity is crucial.

\section{Chemoprevention}

With an increase in cancer morbidity and mortality, the advancement of chemopreventive agents is a key component to the holistic approach in the prevention and treatment of cancer accompanied by fewer adverse side-effects. 'Chemoprevention refers to the use of specific agents (natural or synthetic) to reverse, suppress, or prevent the process of carcinogenesis' (7). These agents target molecules involved in the initiation of apoptosis, such as pro-apoptotic and anti-apoptotic proteins (8). Chemopreventive agents that have exhibited anticancer potential include non-steroidal anti-inflammatory drugs (e.g., sulindac, aspirin and celecoxib) (9), and other FDA-approved drugs, such as Tamoxifen, Raloxifene and HPV vaccines (Cervarix and Gardasil). However, Tamoxifen and Raloxifene have shown adverse side effects in patients (10). Therefore, there is an urgent need for the development of therapeutic agents using compounds that are found naturally, such as phytochemicals in vegetables and fruits, which are lower in toxicity and exhibit little to no adverse side-effects as a result of their use in the prevention and treatment of cancer (11). According to recent studies, a diet rich in fruits and vegetables has been inversely associated with the development of a number of lifestyle-related diseases, such as diabetes and cancer (12-14). However, Petrick et al and the Fukuoka colorectal cancer study in Japan reported no association between the intake of flavonoids and cancer $(15,16)$. Nevertheless, a plethora of studies mention that polyphenols and isothiocyanates have exhibited effectiveness in the prevention of and in reducing the risk of cancer (11-14).

\section{Role of diet in the prevention of carcinogenesis}

Several dietary agents with potential for cancer prevention have been identified and these include polyphenols, isothiocyanates, selenium and allyl compounds (13). These compounds function by controlling biological processes, such as cell proliferation, the induction of programmed cell death, the cell cycle, DNA repair and oxidative stress, affecting cancer pathways in various stages of carcinogenesis (11). The chemosensitivity of natural compounds favors their use as an adjuvant therapy in conventional treatment; however, they can also be utilized exclusively based on their different mechanisms of action against tumor cells (14). Phenolic compounds are secondary metabolites found in plants, that have been extensively studied for their use as treatment and preventive agents. Research highlights the potential of polyphenols to interfere with multiple phases of carcinogenesis. Flavonoids (polyphenolic compounds) are classified into 5 main subclasses as follows: Flavones, flavanols, flavanonols, flavan-3-ols and flavanones. In the present review, the anticarcinogenic properties of flavones are discussed. Notably, in vitro and in vivo studies on dietary flavones have demonstrated their ability to modulate cellular processes, such as cell proliferation, cell cycle arrest and apoptosis (Fig. 1) in various types of cancer (17).

\section{Flavones and apoptosis}

Apoptosis is a crucial process resulting in the removal of undesirable cells within physiological conditions. The process is molecularly characterized by energy-reliant cascade events and morphologically characterized by DNA fragmentation and apoptotic body formation. Apoptosis occurs either via an intrinsic pathway, where the process is activated from signals within the cell, or through an extrinsic pathway where the process is stimulated by death signals received from outside the cell, which are then processed within the cell $(18,19)$ (Fig. 2). A critical characteristic that distinguishes cancer cells from normal cells is their ability to avoid apoptosis. The dysregulation of apoptotic pathways plays a leading role in the onset of carcinogenesis. Therefore, a popular strategy in alternative cancer treatment is the utilization of dietary agents that trigger the apoptosis of cancer cells by modulating apoptotic pathways (18).

Flavones are a subclass of flavonoids which are polyphenolic compounds present in various fruits and vegetables, are known to have antiviral, antioxidant and anticancer activities. Main members of flavones include tangeretin, apigenin, chrysin, nobiletin luteolin and baicalein $(13,14,17)$. Flavones have been broadly studied as anticancer agents and are known to inhibit tumor development in cancer cells by inducing apoptosis. Scientific reports have demonstrated that flavones activate the apoptotic pathway in cancer cells through different mechanisms $(14,17,18)$. The flavones, apigenin, luteolin, baicalein and chrysin, have specifically exhibited shown proteasome-inhibitory effects characterized by decrease in activity the induction of the apoptosis of various cancer cell (17-22). Moreover, flavones have a high safety profile, with no adverse side-effects (19). Studies have elucidated certain biochemical mechanisms through which flavones exert their anticancer effects; these include the following: i) Apoptosis induction $(18,23,24)$; ii) cell cycle arrest at the $\mathrm{G}_{1}$ or $\mathrm{G}_{2} / \mathrm{M}$ phase $(17,23,24)$; iii) inhibition of enzymes involved in metabolism [namely, cytochromes P450 (CYPs)] $(18,26)$; iv) inhibition of reactive oxygen species (ROS) formation by activating phase II metabolizing enzymes $(24,25,27)$; and v) inhibition of vascular endothelial growth factor (VEGF) and basic fibroblast growth factor (bFGF)-mediated angiogenesis $(28,29)$.

In the present review, the anticancer potential of 4 prevalent flavones, namely chrysin, luteolin, baicalein and apigenin, as well as their molecular mechanisms of action that entail their anticancer potential are discussed.

Chrysin. Chrysin, a naturally occurring flavone, is found in honey, Passiflora caerulea (blue passion flower), Oroxylum indicum and bee propolis $(30,31)$. It is specifically a dihydroxyflavone in which the two hydroxy groups are located at positions 5 and 7 (Fig. 3A) and has been investigated for its anti-allergic, anti-inflammatory, antibacterial, antihypertensive, antioxidant and antitumor properties $(13,30,31)$.

Chrysin has been evaluated as a potent anticancer agent and has exhibited tremendous potential against various cancer cell lines, including, breast, bladder, cervical and colorectal cancer. Chrysin has been shown to induce G2/M arrest in SW480 colorectal cancer cells, the inhibition of cyclooxygenase 


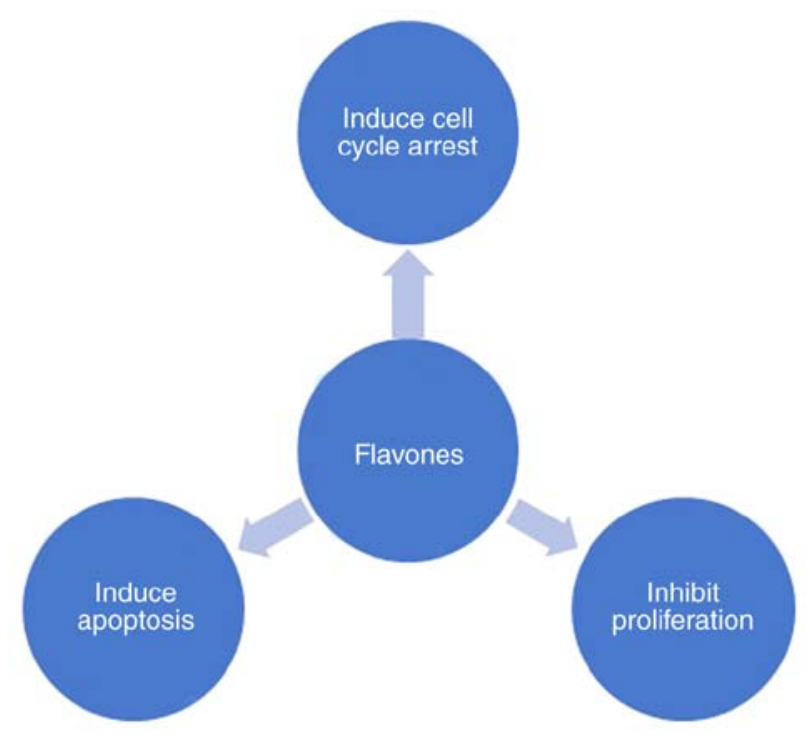

Figure 1. Mechanisms of action of flavones.

(COX-2) via nuclear factor (NF) interleukin (IL)-6 (NF-IL-6), and the fragmentation and apoptosis of $\mathrm{CaCo} 2$ cells (17).

Chrysin induces cytotoxicity, induces apoptosis and inhibits the migration of various cell lines $(30,31)$. The cytotoxic effects of chrysin and the pathways associated with these effects have been elucidated. Certain studies have reported that chrysin induces apoptosis via the intrinsic pathway specifically (30-32), while others have reported that chrysin induces apoptosis via both the intrinsic pathway and the extrinsic pathway $(13,32,33)$. A study on human melanoma cells found that chrysin was able to reduce the viability of the cells in a dose-dependent manner with an IC50 value of 35.8 and $28.3 \mu \mathrm{M}$ in (human uveal melanoma cells) M17 and SP6.5 cell lines, respectively. Further molecular investigation revealed that mitochondrial permeability and cytochrome $c$ levels in the cytosol had increased, and the expression of caspase -3 and -9 was upregulated by chrysin treatment, while the expression of caspase- 8 remained unaltered (30). Concurrently, treatment with chrysin has been shown to activate p53/caspase-9 in hepatocellular carcinoma (HCC); the anti-apoptotic [B-cell lymphoma 2 (Bcl-2)] proteins, including B-cell lymphoma-extra large (Bcl-xL) and Bcl-2 exhibited a decreased expression, while pro-apoptotic proteins exhibited an increased expression; this suggested that chrysin induced apoptosis via the mitochondrial pathway that was associated with the upregulation of caspase-3, BID and $\mathrm{Bcl}-2$-associated $\mathrm{X}$ protein (BAX), and the downregulation of Bcl-2 (34). However, chrysin has been shown to induce the TNF-mediated apoptosis of colorectal cancer, and both the TNF mediated and mitochondria-mediated apoptosis of colon cancer cells $(13,32,33)$. Similarly, another study reported that there was no upregulation of caspase-8, while there was an upregulation of caspase- 9 and -3 , as a result of treatment of bladder cancer cells with chrysin (31). Several other studies have also shown that chrysin treatment induces the apoptosis of human colorectal and HCC cells $(13,33,35)$. These studies have confirmed that chrysin induces the apoptosis of various cell lines by modulating different pathways.

Moreover, it has been reported by a number of studies that the cytotoxic effects of chrysin are mediated via the generation of ROS $(24,31)$. A previous study demonstrated the inhibition of the proliferation of ovarian cancer cells and the induction of cell death by an increase in the concentration of cytoplasmic $\mathrm{Ca}^{2+}$ and ROS levels, as well the loss of mitochondrial membrane potential induced by chrysin treatment (24). Chrysin treatment resulted in the inhibition of AKT/mTOR pathway in triple negative breast cancer and also led to the activation of the tumor necrosis factor (TNF) pathway in multiple cell lines, including lung (A549), rectal (SW837) and colorectal (DLD1 and HCT116) cancer cells by the activation of TNF $\alpha$ and TNF $\beta$ gene expression levels. In HCT116 cells, chrysin induced apoptosis via the mitochondrial pathway; also in A549 human lung adenocarcinoma and HeLa human cancer cell lines chrysin led to tumor necrosis factor-related apoptosis-inducing ligand (TRAIL)-induced apoptosis $(13,32,33)$.

A number of studies have also investigated the anticancer potential of Chrysin in vivo using tumor growth assays/tumor xenografts. As previously reported, the immunohistochemical analysis of tumor tissue exhibited a decreased expression of hexokinase-2 (HK-2) (overexpressed in several types of cancer) following treatment with chrysin, which demonstrated the effect of chrysin on HK-2 in vivo; the reduction of HK-2 in tumor tissue resulted in glycolysis suppression and thus, the supply of energy to maintain tumor growth was blocked, and the tumor cell proliferative ability was weakened (35). A similar study using (human primary glioblastoma cell) U87 xenografts found that 3 weeks of chrysin treatment led to a reduction of tumor weight in mice compared to that of mice delivered treated with refined olive oil as a control; western blot analysis of the tumors revealed that treatment with chrysin blocked tumor xenograft growth via the downregulation of the extracellular signal-regulated kinase (ERK)/nuclear factor E2-related factor 2 (Nrf2) signaling pathway (36). Another study found that chrysin activated Notch1 signaling in an (anaplastic thyroid cancer) ATC xenograft model. The chrysin-treated samples exhibited a moderate to strong expression of Notch1 intracellular domain (NICD), indicating that the apoptosis induced by chrysin was associated with Notch1 activation (37).

Luteolin. Luteolin (3,4,5,7-tetrahydroxy flavone) (Fig. 3B), is a phytochemical ubiquitously found in dietary sources, such as green peppers, olive oil, celery, chamomile tea, peppermint, oregano and broccoli $(38,39)$. Luteolin has been shown to exhibit anti-inflammatory, anti-oxidant and potent cytotoxic activity against various cancer cell lines. It has also been shown to lead to G2 cell cycle arrest and the apoptosis of A549 lung cancer cells via the mitochondrial pathway, whereas it causes either G0/G1 arrest in SH-SY5Y neuroblastoma tumor cells with the loss of mitochondrial potential or G1/S arrest with an increase in the ratio of BAX/Bcl-2 in SMMC-7721 and BEL-7402 (human hepatoma) cells $(13,38)$.

Luteolin has been shown to induce apoptosis by modulating key molecules in both the intrinsic and extrinsic pathways in numerous cancer cell lines, including HT-29, COLO-320 DM, HCT-15, SW480, CaCo-2, HeLa, EC1, KYSE450 and CAOV3/DDP cells (17,39-41). Studies have revealed that luteolin exerts its effects by upregulating the expression of caspase-3/-7/-8, death receptors, TRAIL and Fas/FasL. It also causes inequity in the $\mathrm{Bax} / \mathrm{Bcl}-\mathrm{xL}$ ratio via the inhibition of 


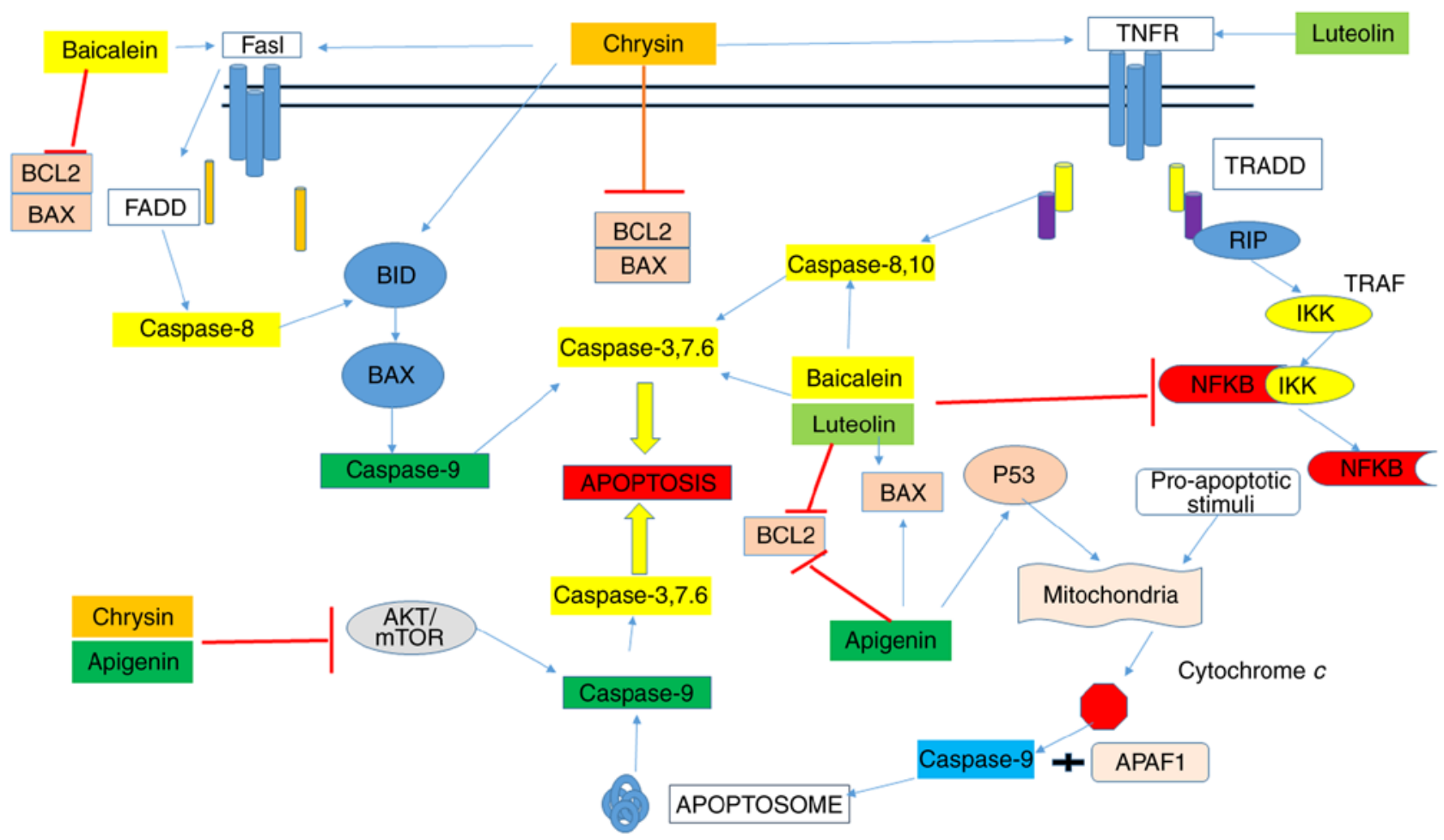

Figure 2. Targets of flavones in intrinsic and extrinsic apoptotic pathways.<smiles>O=c1cc(-c2ccccc2)oc2cc(O)cc(O)c12</smiles><smiles>O=c1cc(-c2ccc(O)c(O)c2)oc2cc(O)cc(O)c12</smiles><smiles>O=c1cc(-c2ccccc2)oc2cc(O)c(O)c(O)c12</smiles><smiles>O=c1cc(-c2ccc(O)cc2)oc2cc(O)cc(O)c12</smiles>

Figure 3. Chemical structures of various flavones. (A) Chrysin, (B) luteolin, (C) baicalein, (D) apigenin.

E6/E7 oncoproteins $(17,40,41)$. Luteolin induces apoptosis by reducing Bcl-2 expression (39) and increasing caspase-3 and caspase- 8 expression (42). It also inhibits NF- $\kappa B$ and activates TNF- $\alpha$-induced apoptosis. Another elucidated mechanism of action is that luteolin promotes the generation of ROS, leading to TNF- $\alpha$-induced apoptosis. ROS generated as a consequence of luteolin treatment have also been shown to activate AMP-activated protein kinase (AMPK), a regulator of NF- $\kappa \mathrm{B}$, which may initiate the cytotoxic effect of luteolin. Furthermore, luteolin induces cytotoxicity to human non-small-cell lung cancer (A549 cells) via the phosphorylation of JNK, and induces apoptosis through the intrinsic pathway, while simultaneously inhibiting NF- $\mathrm{KB}$ (42). Another mechanism of apoptosis induction by luteolin is the accumulation and stabilization of p53; luteolin has been shown to enhance p53 expression in two human colon carcinoma cell lines, CO115 and HCT15 (38).

In addition to this, in vivo studies have found that luteolin reduces tumor weight and volume in mice. Furthermore, Notch1, $\beta$-catenin and Ki-67 expression have been shown to be reduced in tumors from luteolin-treated mice. These results suggest the possibility that luteolin can suppress the progression of gastric cancer via the inhibition of Notch1 expression (43). Another study using human non-small cell lung carcinoma (NCI-H1975) xenograft tumors demonstrated a significant tumor growth inhibition upon the administration of luteolin to mice. Concomitantly, there was no decrease in body weight and no signs of toxicity were manifested following luteolin administration. Immunohistochemical detection displayed that luteolin treatment caused a progressive and significant decrease in the expression of proliferating cell nuclear antigen (PCNA; a cell proliferation marker), CD34 (a microvessel density marker) and epidermal growth factor (EGF) receptors (44).

Baicalein. Baicalein (5,6,7-trihydroxyflavone) (Fig. 3C) is a flavone that was isolated originally from the roots of Scutellaria lateriflora and Scutellaria baicalensis. Baicalein can also be found in thyme and Oroxylum indicum and it has demonstrated to exhibit antioxidant, anti-viral, anti-inflammatory and anticancer properties (45). It has also been shown to exert anticancer effects on HCT116 human colon cancer cells by inhibiting inflammation and inducing apoptosis via the extrinsic pathway and the inactivation of the AKT/PI3K 
pathway; in HT29 cells, it has been shown to induce cell cycle arrest in the $\mathrm{G} 1$ phase, increase the ratio of $\mathrm{BAX} / \mathrm{Bcl}-2$ and to inactivated the AKT/PI3K pathway (17). Its anticancer properties have been attributed to its potent inhibition of several cyclins or cyclin-dependent kinases (CDKs) to regulate the cell cycle, and the downregulation of AKT/mammalian target of rapamycin (mTOR) and MAPK pathways. Both in vitro and in vivo studies have demonstrated that baicalein induces the apoptosis in HT29 colon cancer via the activation of BAX and the downregulation of $\mathrm{Bcl}-2$, and the depolarization of mitochondria; it also inhibits migration via the inhibition of matrix metalloproteinase (MMP)2 and MMP9. The induction of apoptosis and the inhibition of metastasis are mediated via the AKT pathway $(17,45)$. A number of studies have reported the potential of baicalein to induce the apoptosis of various cancer cells, such as HCC (HCCj5), cervical cancer (HeLa) and non-small lung cancer cells; although underlying mechanisms are different between cell lines, overall, apoptosis occurs via the upregulation of the expression of pro-apoptotic proteins, such as cytochrome $c$ and Bax, and the downregulation of the expression of anti-apoptotic proteins, such as Bcl-2. This increases the $\mathrm{Bax} / \mathrm{Bcl}-2$ ratio and triggers the intrinsic apoptotic pathway through the mitochondria, leading to executioner caspase (caspase-3/-9) activation and the cleavage of poly(ADP-ribose) polymerase (PARP) (17,44-46).

Baicalein induces apoptosis by the activation of caspase-9/-3 in breast cancer cells (MDA-MB-231), cervical cancer cells (HeLa and U14) and bladder cancer cells (T24); baicalein induces cell death by upregulating the expression of Bax, caspases and FasL, and downregulating the expression of Bcl-2 (47-49). Similar results were found in HT29colon cancer cells and SGC-7901 gastric cells. In addition, DNA fragmentation was reported in these cell lines. In vivo studies on HT29 colon tumor xenografts verified similar results, while in HCT116 cells, the activation of caspases was observed (45). Another study on MDA-MB-231 cells stated that baicalein considerably diminished the expression of special AT-rich sequence-binding protein-1 (SATB1), SNAIL and vimentin, Wnt1 and $\beta$-catenin proteins, while it augmented the expression of E-cadherin (50). In pancreatic cancer ( $\mathrm{PaCa}$ ) cells, baicalein has been shown to augment the $\mathrm{Bax} / \mathrm{Bcl}-2$ ratio, stimulate the release of mitochondrial cytochrome $c$, and upregulate caspase-3, - 7 and -9 expression. Baicalein and its inhibitory effect on human colon cancer were investigated in vivo and in vitro and the results convey that baicalein exerts a compelling inhibitory effect on HCT-116 cells $(17,50)$. Similarly, by altering $\mathrm{NF}-\kappa \mathrm{B}$ activity, baicalein inhibits cervical cancer cell proliferation as well, and promotes cell apoptosis and can also induce apoptosis by inactivating the PI3K/AKT pathway $(17,46,51)$.

A number of in vivo studies have validated that baicalein exhibits significant anticancer potential in several types of cancer. Upon the gene expression analysis of baicalein-treated $\mathrm{H}-460$ (large cell lung cancer xenograft tumor model) xenografts, it was found that certain genes were differentially regulated in the baicalein-treated group, compared to the control group. The most significant changes were exhibited by genes involved in the DNA damage repair pathway and cell cycle control. The genes ITGB3 (+6.96) and TNFRSF 25 $(+3.4)$, which play a role in the induction of apoptosis, were the most significantly upregulated (52). Another study found that baicalein was able to inhibit the in vivo tumor growth of a breast cancer xenogeneic mouse model by modulating the expression of DNA-damage-inducible transcript 4 (DDIT4), which is responsible for the inhibition of mTOR (53). It has also been demonstrated that baicalein induces the apoptosis of cervical cancer cells by increasing the expression of Bax and decreasing the expression of Bcl-2, which inhibits tumor growth in an in vivo tumor model $(49,51)$. It has also been reported that baicalein downregulates myeloid cell leukemia (Mcl)-1 protein expression, thereby promoting the apoptosis of $\mathrm{PaCa}$ (prostate cancer) cells (45).

Apigenin. Apigenin, a 4',5,7-trihydroxyfavone (Fig. 3D), is abundantly found in fruits and vegetables such as grapes, parsley and apples, and even in beverages such as red wine and chamomile tea $(17,18)$. It aids multiple physiological functions that are beneficial, as it has been shown to exhibit antioxidant, antiviral, anti-inflammatory and antibacterial properties (18). Additionally, there has been ample research conducted on apigenin and its anticancer properties. Several studies have stated that apigenin suppresses the proliferation of various cancer cell lines, including melanoma, hepatic cancer, prostate cancer, lung cancer, colorectal cancer and breast cancer in vitro and in vivo $(11-14,17,18)$, by modulating several biological pathways involved in induction of apoptosis, the inhibition of the cell cycle and the suppression of cell migration and cell invasion. What makes apigenin an effective agent is its ability to trigger apoptosis via both the intrinsic and extrinsic pathways. It achieves this by upregulating the expression of pro-apoptotic proteins, while downregulating that of anti-apoptotic proteins. Research has revealed that it functions by modulating a p53-dependent pathway, which is a pathway related to the induction of apoptosis and cell cycle arrest (28).

More specifically, it has been found that the inhibition of cancer cells by apigenin, specifically colon cancer cells (HT-29, SW480, HCT-116 and CaCo-2) occurs through the downregulation of $\mathrm{Cdc}-2$, cyclin $\mathrm{B} 1$ and $\mathrm{Cdc}-25$, and the upregulation of p21 and p53. Further investigation revealed that cells treated with apigenin exhibited an accumulation in the G2/M phase of the cell cycle, whereas in HT29 cells exhibiting mutant p53 exhibited the induction of p38 and ERK, and the downregulation of mTOR and cyclin D1 (17). A similar study using pancreatic cells (with p53 gene mutations) indicated that apigenin induced cell cycle arrest followed by apoptotic cell death through p53-related pathways (54). In colon adenocarcinoma ( $\mathrm{SW} 480$ ) cells, apigenin was shown to increase the expression of caspases and Bax, and inhibit Bcl-2 expression (17). The treatment of bladder carcinoma cells with apigenin activated the intrinsic mitochondrial pathway marked by the release of cytochrome $c$, the stimulation of Bax, PARP and caspases, and the inhibition of Bcl-2 (53). It has also been reported that apigenin amplifies the production of ROS, leading to cytotoxicity in all cervical cancer cell lines ( $\mathrm{SiHa}$, HeLa, C33A and CaSki (27). In a previous study, apigenin induced WAF1/p21 (cell cycle inhibitor), p53 protein, caspase-3 activation. Furthermore, apoptosis was indicated by DNA fragmentation in these cells (55). Another study using colon cancer cells demonstrated the induction of apoptosis by apigenin via 
the inhibition of the phosphorylation of signal transducer and activator of transcription 3 (STAT3) and the downregulation Bcl-xL and Mcl-1, which are anti-apoptotic (56). Another similar study demonstrated that in malignant mesothelioma cells, apigenin induced an increase in the $\mathrm{Bax} / \mathrm{Bcl}-2$ ratio, $\mathrm{p} 53$ expression and the subsequent stimulation of caspases -9 and -8 led to apigenin-induced apoptosis, which was marked by the cleavage of PARP (57). It was also demonstrated by another study that apoptosis was induced by apigenin in cholangiocarcinoma via a caspase-dependent pathway (58). Normal cells have not exhibited any significant cytogenotoxic effects upon apigenin treatment; this specificity is associated with its low toxicity indicate its potential as natural chemopreventive agent (59).

The anticancer potential of apigenin has also been explored in several in vivo models. Studies have revealed that apigenin inhibits colorectal cancer induced by azoxymethane (AOM) in Sprague-Dawley (SD) rats and that apigenin decreases tumor volume in human prostate cancer (PC-3). Treatment of tumors with apigenin was also shown to result in an increase in the apoptotic proportion of cells in the tumor. More importantly, apigenin intake did not seem to reduce body weight and appetite in the animals $(17,60)$. Another similar in vivo study reported that apigenin modulated the $\mathrm{PI} 3 \mathrm{~K} / \mathrm{AKT} / \mathrm{FoxO}$-signaling pathway, restricting the process of tumorigenesis in transgenic adenocarcinoma of mouse prostate (TRAMP) mice (61). Furthermore, apigenin intake also led to tumor growth inhibition in U937 (human leukemia cells) xenografts. This was accompanied by the inhibition of AKT and induction of JNK (57).

Although flavones are potential anticancer agents, some investigators have identified the low bioavailability and instability (enzymes, acid, interference by other nutrients) of these compounds inside the gut during the processing of food in the digestive system. Thus, the best method recommended thus far is delivery of these agents via nanoparticles $(14,20)$.

\section{Conclusion and future prospects}

Cancer is a multifactorial and multi-step process characterized by a variety of hallmarks, with uncontrolled proliferation and resistance to apoptosis being the most important. Cancer positions among the most domineering medical issues affecting the human population, and chemopreventive approaches signify a hopeful strategy with which to prevent occurrence and death. Flavones, as natural compounds, may elicit great variability in their therapeutic results. Although a number of in vitro studies have been conducted, clinical trials using specific concentrations of these agents are underway. Furthermore, experimental and clinical studies focused on flavones need to be performed in order to clarify the value of these molecules in cancer treatment. Although ample data have been collected, further investigations are warranted to investigate the use of flavones as a treatment option, taking into consideration the proper delivery system of these agents for clinical settings.

\section{Acknowledgements}

Not applicable.

\section{Funding}

No funding was received.

\section{Availability of data and materials}

All data generated or analyzed during this study are included in this published article or are available from the corresponding author on reasonable request.

\section{Authors' contributions}

AH conceptualized the study and designed the study content and figures, and was also involved in the editing of the manuscript. RR was involved in the writing of the manuscript, in the literature search and in the processing of the figures/images. RS was involved in data collection. All authors have read and approved the final manuscript.

\section{Ethics approval and consent to participate}

Not applicable.

\section{Patient consent for publication}

Not applicable.

\section{Competing interests}

The authors declare that they have no competing interests.

\section{References}

1. Gelbart WM, Lewontin RC, Wessler SR, Suzuki DT, Miller JH and Griffiths AJF: An Introduction to Genetic Analysis, 8th edition. W.H.Freeman \& Co Ltd, 2004.

2. Bray F, Ferlay J, Soerjomataram I, Siegel RL, Torre LA and Jemal A: Global cancer statistics 2018: GLOBOCAN estimates of incidence and mortality worldwide for 36 cancers in 185 countries. CA Cancer J Clin 68: 394-424, 2018.

3. Arem $\mathrm{H}$ and Loftfield $\mathrm{E}$ : Cancer epidemiology: A survey of modifiable risk factors for prevention and survivorship. Am J Lifestyle Med 12: 200-210, 2017.

4. Theodoratou E, Timofeeva M, Li X, Meng X and Ioannidis JPA: Nature, nurture, and cancer risks: Genetic and nutritional contributions to cancer. Annu Rev Nutr 37: 293-320, 2017.

5. Wu S, Zhu W, Thompson P and Hannun YA: Evaluating intrinsic and non-intrinsic cancer risk factors. Nat Commun 9: 3490, 2018.

6. Tripathi KD: Essentials of Medical Pharmacology, 7th edition. Jaypee Brothers Medical Publishers (P) Ltd, 2013.

7. Steward WP and Brown K: Cancer chemoprevention: A rapidly evolving field. Br J Cancer 109: 17, 2013.

8. Aggarwal BB, Takada Y and Oommen OV: From chemoprevention to chemotherapy: Common targets and common goals. Expert Opin Investig Drugs 13: 1327-1338, 2004.

9. Patterson SL, Colbert Maresso K and Hawk E: Cancer chemoprevention: Successes and failures. Clin Chem 59: 94-101, 2013.

10. Sauter ER: Breast cancer prevention: current approaches and future directions. Eur J Breast Health 14: 64-71, 2018.

11. de Melo FHM, Oliveira JS, Sartorelli VOB and Montor WR: Cancer chemoprevention: Classic and epigenetic mechanisms inhibiting tumorigenesis. What have we learned so far? Front Oncol 8: 644, 2018.

12. Heller MC, Keoleian GA and Willett WC: Toward a life cycle-based, diet-level framework for food environmental impact and nutritional quality assessment: A critical review. Environ Sci Technol 47: 12632-12647, 2013.

13. Zhou Y,Zheng J, Li Y, Xu DP, Li S, Chen YM and Li HB: Natural polyphenols for prevention and treatment of cancer. Nutrients 8: E515, 2016. 
14. Costea T, Hudită A, Ciolac OA, Gălăteanu B, Ginghină O, Costache M, Ganea C and Mocanu MM: Chemoprevention of colorectal cancer by dietary compounds. Int J Mol Sci 19: E3787, 2018.

15. Petrick JL, Steck SE, Bradshaw PT, Trivers KF, Abrahamson PE, Engel LS, He K, Chow WH, Mayne ST, Risch HA, et al: Dietary intake of flavonoids and oesophageal and gastric cancer: Incidence and survival in the United States of America (USA). Br J Cancer 112: 1291-1300, 2015.

16. Wang ZJ, Ohnaka K, Morita M, Toyomura K, Kono S, Ueki T, Tanaka M, Kakeji Y, Maehara Y, Okamura T, et al: Dietary polyphenols and colorectal cancer risk: The Fukuoka colorectal cancer study. World J Gastroenterol 19: 2683-2690, 2013.

17. Koosha S, Alshawsh MA, Looi CY, Seyedan A and Mohamed Z: An association map on the effect of flavonoids on the signaling pathways in colorectal cancer. Int J Med Sci 13: 374-385, 2016.

18. Yan X, Qi M, Li P, Zhan Y and Shao H: Apigenin in cancer therapy: Anti-cancer effects and mechanisms of action. Cell Biosci 7: 50, 2017

19. Lin $\mathrm{CH}$, Chang CY, Lee KR, Lin HJ, Chen TH and Wan L: Flavones inhibit breast cancer proliferation through the Akt/FOXO3a signaling pathway. BMC Cancer 15: 958, 2015.

20. Amawi H, Ashby CR Jr and Tiwari AK: Cancer chemoprevention through dietary flavonoids : What's limiting? Chin J Cancer 36 $50,2017$.

21. Moga MA, Dimienescu OG, Arvatescu CA, Mironescu A, Dracea L and Ples L: The role of natural polyphenols in the prevention and treatment of cervical cancer-an overview. Molecules 21: E1055, 2016.

22. Landis-Piwowar KR, Milacic V and Dou QP: Relationship between the methylation status of dietary flavonoids and their growth-inhibitory and apoptosis-inducing activities in human cancer cells. J Cell Biochem 105: 514-523, 2008

23. Ryu S, Lim W, Bazer FW and Song G: Chrysin induces death of prostate cancer cells by inducing ROS and ER stress. J Cell Physiol 232: 3786-3797, 2017.

24. Lim W, Ryu S, Bazer FW, Kim SM and Song G: Chrysin attenuates progression of ovarian cancer cells by regulating signaling cascades and mitochondrial dysfunction. J Cell Physiol 233: 3129-3140, 2018

25. Granato M, Gilardini Montani MS, Santarelli R, D'Orazi G Faggioni A and Cirone M: Apigenin, by activating p53 and inhibiting STAT3, modulates the balance between pro-apoptotic and pro-survival pathways to induce PEL cell death. J Exp Clin Cancer Res 36: 167, 2017.

26. Romagnolo DF, Donovan MG, Papoutsis AJ, Doetschman TC and Selmin OI: Genistein prevents BRCA1 CpG methylation and proliferation in human breast cancer cells with activated aromatic hydrocarbon receptor. Curr Dev Nutr 1: e000562, 2017.

27. Souza RP, Bonfim-Mendonça PS, Gimenes F, Ratti BA, Kaplum V Bruschi ML, Nakamura CV, Silva SO, Maria-Engler SS and Consolaro ME: Oxidative stress triggered by apigenin induces apoptosis in a comprehensive panel of human cervical cancer-derived cell lines. Oxid Med Cell Longev 2017: 1512745 , 2017.

28. Sung B, Chung HY and Kim ND: Role of apigenin in cancer prevention via the induction of apoptosis and autophagy. J Cancer Prev 21: 216-226, 2016.

29. Ci Y, Qiao J and Han M: Molecular mechanisms and metabolomics of natural polyphenols interfering with breast cancer metastasis. Molecules 21: E1634, 2016.

30. Xue C, Chen Y, Hu DN, Iacob C, Lu C and Huang Z: Chrysin induces cell apoptosis in human uveal melanoma cells via intrinsic apoptosis. Oncol Lett 12: 4813-4820, 2016.

31. Xu Y, Tong Y, Ying J, Lei Z, Wan L, Zhu X, Ye F, Mao P, Wu X, Pan R, et al: Chrysin induces cell growth arrest, apoptosis, and ER stress and inhibits the activation of STAT3 through the generation of ROS in bladder cancer cells. Oncol Lett 15 9117-9125, 2018.

32. Chylińska-Wrzos P, Lis-Sochocka M and Jodlowska-Jedrych B: Chrysin and its potential antineoplastic effect. Eur J Biol Res 7: 245-254, 2017.

33. Ronnekleiv-Kelly SM, Nukaya M, Díaz-Díaz CJ, Megna BW, Carney PR, Geiger PG and Kennedy GD: Aryl hydrocarbon receptor-dependent apoptotic cell death induced by the flavonoid chrysin in human colorectal cancer cells. Cancer Lett 370: 91-99, 2016.

34. Zhang Q, Ma S, Liu B, Liu J, Zhu R and Li M: Chrysin induces cell apoptosis via activation of the $\mathrm{p} 53 / \mathrm{Bcl}-2 /$ caspase-9 pathway in hepatocellular carcinoma cells. Exp Ther Med 12: 469-474, 2016.
35. Xu D, Jin J, Yu H, Zhao Z, Ma D, Zhang C and Jiang H: Chrysin inhibited tumor glycolysis and induced apoptosis in hepatocellular carcinoma by targeting hexokinase-2. J Exp Clin Cancer Res 36: 44, 2017

36. Wang J, Wang H, Sun K, Wang X, Pan H, Zhu J, Ji X and Li X: Chrysin suppresses proliferation, migration, and invasion in glioblastoma cell lines via mediating the ERK/Nrf2 signaling pathway. Drug Des Devel Ther 12: 721-733, 2018.

37. Yu XM, Phan T, Patel PN, Jaskula-Sztul R and Chen H: Chrysin activates Notch1 signaling and suppresses tumor growth of anaplastic thyroid carcinoma in vitro and in vivo. Cancer 119: 774-781, 2013.

38. Tuorkey MJ: Molecular targets of luteolin in cancer. Eur J Cancer Prev 25: 65-76, 2016

39. Ham S, Kim KH, Kwon TH, Bak Y, Lee DH, Song YS, Park SH, Park YS, Kim MS, Kang JW, et al: Luteolin induces intrinsic apoptosis via inhibition of E6/E7 oncogenes and activation of extrinsic and intrinsic signaling pathways in HPV-18-associated cells. Oncol Rep 31: 2683-2691, 2014.

40. Chen P, Zhang JY, Sha BB, Ma YE, Hu T, Ma YC, Sun H, Shi JX, Dong ZM and Li P: Luteolin inhibits cell proliferation and induces cell apoptosis via down-regulation of mitochondrial membrane potential in esophageal carcinoma cells EC1 and KYSE450. Oncotarget 8: 27471-27480, 2017.

41. Wang H, Luo Y, Qiao T, Wu Z and Huang Z: Luteolin sensitizes the antitumor effect of cisplatin in drug-resistant ovarian cancer via induction of apoptosis and inhibition of cell migration and invasion. J Ovarian Res 11: 93, 2018.

42. Liao Y, Xu Y, Cao M, Huan Y, Zhu L, Jiang Y, Shen W and Zhu G: Luteolin induces apoptosis and autophagy in mouse macrophage ANA-1 cells via the Bcl-2 pathway. J Immunol Res 2018: 4623919, 2018.

43. Zang M, Hu L, Fan ZY, Wang HX, Zhu ZL, Cao S, Wu XY, Li JF, Su LP, Li C, et al: Luteolin suppresses gastric cancer progression by reversing epithelial-mesenchymal transition via suppression of the Notch signaling pathway. J Transl Med 15: 52, 2017.

44. Hong Z, Cao X, Li N, Zhang Y, Lan L, Zhou Y, Pan X, Shen L, Yin $Z$ and Luo L: Luteolin is effective in the non-small cell lung cancer model with L858R/T790M EGF receptor mutation and erlotinib resistance. Br J Pharmacol 171: 2842-2853, 2014.

45. Liu H, Dong Y, Gao Y, Du Z, Wang Y, Cheng P, Chen A and Huang H: The fascinating effects of baicalein on cancer: A review. Int J Mol Sci 17: E1681, 2016.

46. Wang Y, Xia J, Tang X, Tang L, Mao X, Zhang Y and Yu X: Baicalein promotes the apoptosis of HeLa cells by inhibiting ERK1/2 expression. Xi Bao Yu Fen Zi Mian Yi Xue Za Zhi J 32: 1507-1512, 2016 (In Chinese).

47. Choi EO, Park C, Hwang HJ, Hong SH, Kim GY, Cho EJ, Kim WJ and Choi YH: Baicalein induces apoptosis via ROS-dependent activation of caspases in human bladder cancer 5637 cells. Int J Oncol 49: 1009-1018, 2016

48. Yan W, Ma X, Zhao X and Zhang S: Baicalein induces apoptosis and autophagy of breast cancer cells via inhibiting PI3K/AKT pathway in vivo and vitro. Drug Des Devel Ther 12: 3961-3972, 2018.

49. Peng Y, Guo C, Yang Y, Li F, Zhang Y, Jiang B and Li Q Baicalein induces apoptosis of human cervical cancer HeLa cells in vitro. Mol Med Rep 11: 2129-2134, 2015.

50. Ma X, Yan W, Dai Z, Gao X, Ma Y, Xu Q, Jiang J and Zhang S: Baicalein suppresses metastasis of breast cancer cells by inhibiting EMT via downregulation of SATB1 and Wnt/ $\beta$-catenin pathway. Drug Des Devel Ther 10: 1419-1441, 2016.

51. Yu X, Liu Y, Wang Y, Mao X, Zhang Y and Xia J: Baicalein induces cervical cancer apoptosis through the NF- $\kappa \mathrm{B}$ signaling pathway. Mol Med Rep 17: 5088-5094, 2018.

52. Cathcart MC, Useckaite Z, Drakeford C, Semik V, Lysaght J, Gately K, O'Byrne KJ and Pidgeon GP: Anti-cancer effects of baicalein in non-small cell lung cancer in-vitro and in-vivo. BMC Cancer 16: 707, 2016.

53. Wang Y, Han E, Xing Q, Yan J, Arrington A, Wang C, Tully D, Kowolik CM, Lu DM, Frankel PH, et al: Baicalein upregulates DDIT4 expression which mediates mTOR inhibition and growth inhibition in cancer cells. Cancer Lett 358: 170-179, 2015.

54. King JC, Lu QY, Li G, Moro A, Takahashi H, Chen M, Go VL, Reber HA, Eibl G and Hines OJ: Evidence for activation of mutated p53 by apigenin in human pancreatic cancer. Biochim Biophys Acta 1823: 593-604, 2012.

55. Shi MD, Shiao CK, Lee YC and Shih YW: Apigenin, a dietary flavonoid, inhibits proliferation of human bladder cancer T-24 cells via blocking cell cycle progression and inducing apoptosis. Cancer Cell Int 15: 33, 2015. 
56. Maeda Y, Takahashi H, Nakai N, Yanagita T, Ando N, Okubo T, Saito K, Shiga K, Hirokawa T, Hara M, et al: Apigenin induces apoptosis by suppressing Bcl-xl and Mcl-1 simultaneously via signal transducer and activator of transcription 3 signaling in colon cancer. Int J Oncol 52: 1661-1673, 2018.

57. Masuelli L, Benvenuto M, Mattera R, Di Stefano E, Zago E, Taffera G, Tresoldi I, Giganti MG, Frajese GV, Berardi G, et al: In vitro and in vivo anti-tumoral effects of the flavonoid apigenin in malignant mesothelioma. Front Pharmacol 8: 373, 2017.

58. Subhasitanont P, Chokchaichamnankit D, Chiablaem K, Keeratichamroen S, Ngiwsara L, Paricharttanakul NM, Lirdprapamongkol K, Weeraphan C, Svasti J and Srisomsap C: Apigenin inhibits growth and induces apoptosis in human cholangiocarcinoma cells. Oncol Lett 14: 4361-4371, 2017.

59. Vrhovac Madunic I, Madunić J, Antunović M, Paradžik M, Garaj-Vrhovac V, Breljak D, Marijanović I and Gajski G: Apigenin, a dietary flavonoid, induces apoptosis, DNA damage, and oxidative stress in human breast cancer MCF-7 and MDA MB-231 cells. Naunyn Schmiedebergs Arch Pharmacol 391: 537-550, 2018
60. Shukla S, Kanwal R, Shankar E, Datt M, Chance MR, Fu P, MacLennan GT and Gupta S: Apigenin blocks IKK $\alpha$ activation and suppresses prostate cancer progression. Oncotarget 6 : 31216-31232, 2015.

61. Shukla S, Bhaskaran N, Babcook MA, Fu P, Maclennan GT and Gupta S: Apigenin inhibits prostate cancer progression in TRAMP mice via targeting PI3K/Akt/FoxO pathway. Carcinogenesis 35: 452-460, 2014.

This work is licensed under a Creative Commons Attribution-NonCommercial-NoDerivatives 4.0 International (CC BY-NC-ND 4.0) License. 